\title{
A stress point algorithm for an elastoplastic model in unsaturated soils
}

\author{
J. Vaunat*, J.C. Cante, A. Ledesma, A. Gens \\ Technical University of Catalonia (UPC), Barcelona, Spain
}

Received in final revised form 5 May 1999

\begin{abstract}
Two stress fields, combination of total stresses, liquid pressure and gas pressure have to be considered to explain the deformational behaviour of unsaturated media. Elastoplastic models developed for these materials consider generally two yield surfaces, each one associated to a stress field, and whose intersection produces a corner in the space of generalised stress components. In this paper, a stress point algorithm is proposed to cope with the integration of such constitutive laws, which can be seen as non smooth multisurface plastic models in the space of the two stress fields. The basic model developed by Alonso et al. (Alonso, E.E., Gens, A., 1990. A constitutive model for partially saturated soils. Géotechnique 40 (3), 405-430), which will be used to test the algorithm, is first described. Generalised stress and strain variables are then defined. Implementation of the return mapping algorithm, based on an implicit integration scheme, is presented with special attention devoted to the problem of mixed control imposed by the F.E. formulation generally used to analyse the hydromechanical behaviour of unsaturated media. Validation results on distinct generalised stress paths are given at the end. (C) 2000 Published by Elsevier Science Ltd. All rights reserved.
\end{abstract}

Keywords: A. Plastic collapse; B. Geological material; C. Finite elements; C. Numerical algorithm; Unsaturated soils

\section{Introduction}

Unsaturated soils are triphasic porous media where gas phase (usually air), liquid phase (usually water) and solid phase (soil grains) exist at dissident points of the space. As a consequence of the complexity of the spatial distribution of each phase

\footnotetext{
* Corresponding author. Tel.: + 34-93-401-7248; fax: + 34-93-401-7251.

E-mail address: jean.vaunat@upc.es (J. Vaunat).
} 
and of the interactions existing between them, description of flow and transport in such media is generally carried out by considering overlapped substitute continua over the entire space, each one corresponding to a given phase. Three main strategies exist to construct these continua. The first one considers macroscopic balance equations based generally on mixture theory and looks for phenomenological constitutive laws at that scale, restricted by the entropy inequality in order not to violate the second law of thermodynamics (Coussy, 1995; Alcoverro et al., 1998). Formulations given by Olivella (1995) and Thomas and He (1995) belong to this framework. The second one starts from microscopical balance equations and performs a scale change by averaging techniques, that allows interpretation of the averaged variables in terms of their microscopic counterparts (Hassanizadeh and Gray 1979a,b, 1980; Murad, 1997; Lewis and Schrefter, 1998). In this strategy, called hybrid mixture theory, constitutive laws are also developed at the averaged scale by exploiting the entropy inequality. The third strategy consists in passing both microscopic balance equations and constitutive relationships at the macroscopic scale by homogenization techniques. The work of Chateau and Dormieux (1995) is an example of this approach.

In case of isothermal problems, mass and momentum balance equations of each phase have to be solved. By use of the first strategy, assuming that the processes

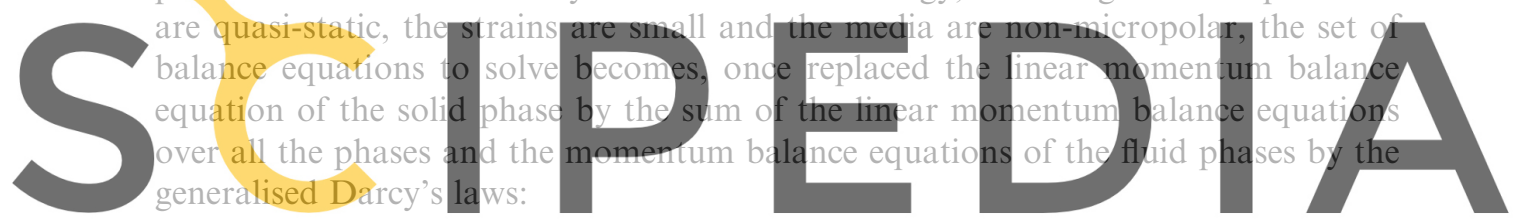

Register for free at https//WwW.Scipedia.com to download the version without the watermark

Water mass balance

Air mass balance

$$
\begin{aligned}
& \frac{D^{s} \rho^{w} n S_{v}^{w}}{D t}+\nabla\left(\rho^{w} \mathbf{q}_{v}^{w}\right)+\rho^{w} n S_{v}^{w} \nabla \mathbf{v}^{s}+f^{w}=0 \\
& \frac{D^{s} \rho^{a} n S_{v}^{a}}{D t}+\nabla\left(\rho^{a} \mathbf{q}_{v}^{a}\right)+\rho^{a} n S_{v}^{a} \nabla \mathbf{v}^{s}+f^{a}=0
\end{aligned}
$$

where $\boldsymbol{\sigma}$ is the (symmetric) total stress tensor, $\mathbf{b}$ the volumetric force vector of the mixture, $D^{s} / D t$ the time derivative with respect to the solid phase, $\rho^{\phi}(\phi=w-$ for water, $a$ - for air) the density of phase $\phi, n$ the porosity, $s_{v}^{\phi}$ the volume of phase $\phi$ over the volume of all the fluid phases, $\mathbf{q}_{v}^{\phi}=n S_{v}^{\phi}\left(\mathbf{v}^{\phi}-\mathbf{v}^{s}\right)$ the Darcy's velocity of fluid phase $\phi, \mathbf{v}^{\phi}$ the velocity of phase $\phi$ and $f^{\phi}$ a mass source/sink term of phase $\phi$. The soil mechanics convention (compression stresses and strains are positive) is used and will be followed in all the paper. By applying the principle of virtual work, the weak form of Eq. (1) is obtained, usually solved by Finite Element (F.E.) method using a standart Galerkin discretisation in space. These formulations consider generally solid displacements, air pressure and water pressure as main unknowns (Schreller and Zhan Xiaoyong, 1993; Olivella, 1995; Thomas and He, 1995).

Description of the mechanical behaviour requires the definition of a constitutive relationship between the strains and the stresses acting on the solid phase. When focused on a phenomenological point of view, this relation is generally established 
between macroscopical variables measurable during experimental tests, usually $\sigma_{i j}, p^{a}$ (air pressure) and $p^{w}$ (water pressure). Because of difficulties in finding a single stress concept able to explain the behaviour of unsaturated soils, the acceptance of considering two effective stress fields in these media progressively emerged. Contributions from Bishop and Donald (1961), Matyas and Radhakrishna (1968) and Fredlund and Morgenstern (1977) led to a formal proposition of any pair of stress fields among the net stresses $\sigma_{i j}^{U}=\sigma_{i j}-\delta_{i j} p^{a}\left(\delta_{i j}\right.$ being the Kronecker delta), the effective stresses $\sigma_{i j}^{l}=\sigma_{i j}-\delta_{j i} p^{w}$ and the suction $p^{a}-p^{w}$. The existence of these two stress fields has an intuitive meaning in granular media, where the deformational response of the skeleton depends not only on contact forces between grains and pore filling fluid pressures, but also on forces exerted on the skeleton by the interfaces existing between the wetting and non-wetting phases. By use of the hybrid mixture theory, Hassanizadch and Gray (1990) formalised a different double stress concept based on the definition of the effective stresses of the solid phase and on the capillary pressure, this later related to the net release of energy by the phases and interfaces in the system. From this work, Lewis and Schrefier (1998) proposed to use the effective stresses defined as $\sigma_{i j}^{\text {eff }}=\sigma_{i j}-\delta_{i j} S_{v}^{a} p^{a}-\delta_{i j} S_{v}^{w} p^{w}$ and the suction. In any case, whatever the concepts considered, two stress fields, weighted combinations of total stresses, water pressure and air pressure have generally to be considered to deal with

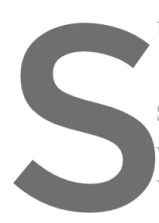
the mechanical resp

Recent advances h

soils under stress and

work of hardening

Wheeler and Sivakumar
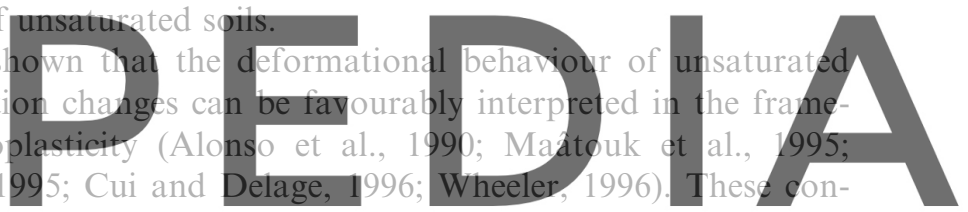

stitutive laws generally extend elastoplastic models developed for saturated soils to

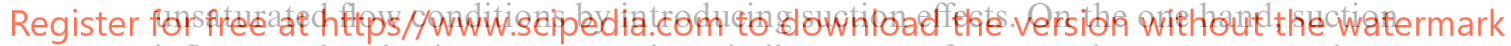
influences the plastic parameters, in a similar way as, for example, temperature does. On the other hand, change in suction produces deformation. The first effect is of general acceptance and forms the background of all elastoplastic models for unsaturated geomaterials. The physical meaning of the second phenomenon is a matter of discussion and its inclusion into the distinct models will generally differ depending on the stress concept considered in unsaturated conditions. Any general implementation procedure of elastoplastic models for unsaturated soils cannot be thus presently contemplated.

Among all the elastoplastic relationships for unsaturated media, the pioneering model developed by Alonso et al. (1990) remains to date a reference work. Based on an extension of the modified Cam clay model (Roscoe and Burland, 1968), it gives a simple representation of the behaviour of unsaturated soils, by use of relatively few parameters, most of them directly deducible from laboratory tests. The main features of the model are:

1. to work with net stresses and suction as stress variables,

2. to consider an independent elastic strain component associated to suction (the elastic strain increment is then split into the elastic strain increment due to net stress changes and the elastic strain increment due to suction changes), 
3. to close the elastic domain in the net stress-suction space by two yield surfaces, whose intersection produces a corner.

These features require a specific treatement for the F.E. implementation of the elastoplastic law. Implementation of strategies to integrate rate independent elastoplasticity relations has been the subject of numerous works (Simo and Taylor, 1985; Ortiz and Simo, 1986; Sloan, 1987; Crisfield, 1991; Chaboche and Cailletaud, 1996, among others) in part devoted to the respective merits of explicit and implicit integration schemes. More specifically, Gens and Potts (1988) compared the efficiency of distinct explicit methods combined with implicit return mapping algorithms in order to cope with integration of critical state models for soils. As concerns the implicit integration scheme, Runesson (1987) underlined its sound mathematical basis based on convex analysis and its conceptual elegance as the result of a consistent evaluation of hardening law, flow rule and derivatives of yield surface. He gave the formulation for the closest point projection method (CPPM) in the case of modified Cam clay model. For the same model, Borja and Lee (1990) and Borja (1991) presented the numerical implementation of CPPM coupled with a non linear elastic predictor and highlighted its good performance. More recently, Ledesma et al. (1995) and Li and Charlier (1995) presented a F.E. implementation of the model of

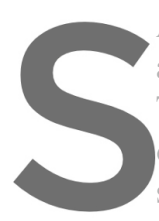
Alonso et al. (1990)
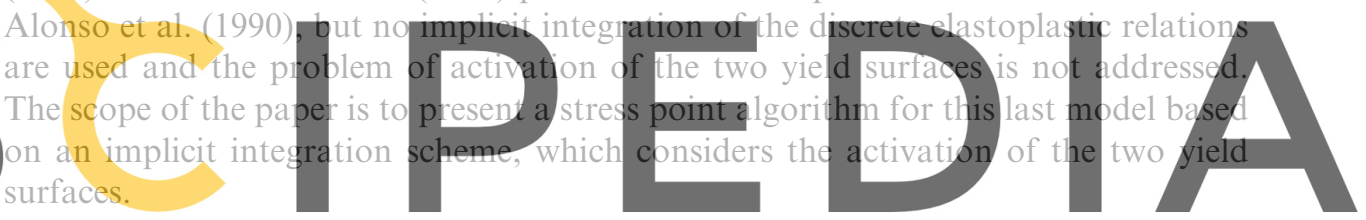

If a generalised stress is defined by merging in one vector the suction and all the

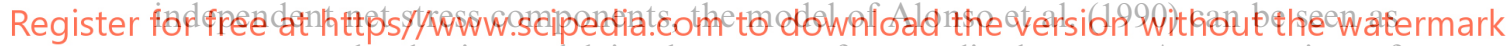
a non smooth plastic model in the space of generalised stress. An extension of CPPM to the case of non smooth multisurface plasticity in the classical stress-strain space was presented by Simo et al. (1988). The algorithm derived by these authors can be extended to unsaturated conditions by expressing elastoplastic relations in terms of generalised stress. To keep the structure of the procedure, it is, however, necessary to consider a new strain component associated to suction in order to build a generalised strain vector of the same length as the generalised stress vector. Such artifice to further the extension of the algorithm of Simo et al. (1988) to unsaturated conditions is described in the paper. The authors do not pretend to justify the physical basis of the generalised variable. As seen before, the primary variables considered in the F.E. formulation are solid displacements, water pressure and air pressure. The variables given as input of the mechanical constitutive subroutine are then increment of total strain and increment of suction. The stress point algorithm developed should then work with a mixed generalised stress-generalised strain control. Tangent formulations of the constitutive relations in plasticity under mixed control in the classical stress-strain space have been presented by Klisinski et al. (1992) and Mróz and Rodzik (1995). Algorithmic procedures to integrate the rate equations for a finite increment of mixed stress-strain vector were presented by Alawaji et al. (1990, 1992). A hybrid procedure has been adapted to the present model. 
In the first part of the paper, the model of Alonso et al. (1990) is briefly reviewed. This model will be referred as Barcelona basic model. Generalised variables are then presented. Afterwards, evolution equations of the model are reformulated in terms of the generalised variables previously defined. In a fourth part, integration of the discrete form of these equations is presented and mixed control introduced. Finally, validation and performance tests on distinct generalised stress and strain paths are given.

\section{Barcelona basic model for unsaturated media}

Barcelona basic model (BBM) is an extension of modified Cam clay model for unsaturated conditions. Both models will be briefly recorded, using the Soil Mechanics convention.

Modified Cam clay model (MCCM) is a critical state based model initially developed for saturated soils (Roscoe and Burland, 1968) which relates the strains to the changes in effective stresses $\sigma_{i j}^{\prime}=\sigma_{i j}-\delta_{i j} p^{w}$. Equations and parameters of the model are described in Table 1. $p^{\prime}$ is the mean effective stress and $J$ the square root of the second invariant of deviatoric stress tensor. Lode angle is considered constant and

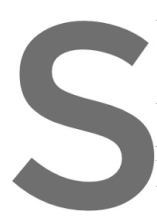
the non associated pra

\section{The yield locus in th}

tered on the hydrost

metric plastic strain is

the material is contract
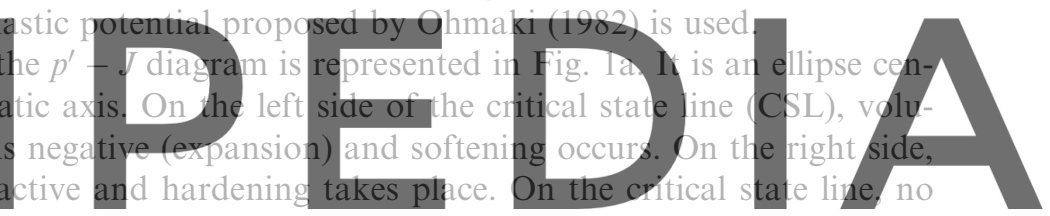

volumetric plastic strain takes place and the stresses reach steady-state values. This

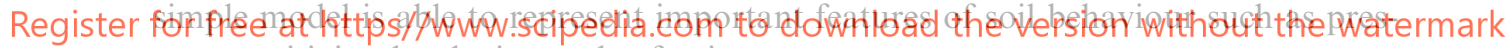
sure sensitivity, hardening and softening response.

The model works with five parameters, identifiable by loading/unloading and shearing tests. They are illustrated in Fig. 1a. $\kappa$ is equal to the slope of the line in the $e-\ln \left(p^{\prime}\right)$ diagram during isotropic elastic loading ( $e$ is the void ratio defined as the volume of pores over the volume of solid phase). $G$ is the shear modulus, considered constant. $p_{0}^{*}$ is a history variable equal to the highest mean effective stress known by the material. $M$ is the slope of the critical state line and is related to the friction angle of the material. $\lambda$ is the slope of the line in the $e-\ln \left(p^{\prime}\right)$ diagram during isotropic plastic loading. More details on the model and the identification of the parameters can be found in Wood (1990).

MCCM also has crucial limitations, among them disregards for anisotropy, intermediate stress influence, occurrence of irrecoverable strain inside the yield surface, realistic shear strength on the softening side and strain rate effect. However, it provides a good qualitative understanding of the behaviour of cohesive materials.

In the extension proposed by Alonso et al. (1990), the strains are related to changes in net stresses $\sigma_{i j}^{\prime \prime}=\sigma_{i j}-\delta_{i j} p^{a}$ and in suction $p^{a}-p^{w}$. Equations and parameters of the BBM model are described in Table 2. $p^{\prime \prime}$ denotes the net mean stress and $p_{\mathrm{atm}}$ the atmospheric pressure.

In this model, a change in suction produces a volumetric elastic strain. The yield function and the critical state line change with suction. This dependency can be 
Table 1

Modified Cam clay model with constant Lode angle and Ohmaki's plastic potential

Equations Parameters

Elastic part

$$
\begin{aligned}
& \mathrm{d} \varepsilon_{i j}^{e}=\frac{2 G-3 K}{6 K G} \mathrm{~d} p^{\prime} \delta_{i j}+\frac{1}{2 G} \mathrm{~d} \sigma_{i j}^{\prime} \quad \kappa, G \\
& K=\frac{(1+e) p^{\prime}}{\kappa}, G=\mathrm{constant}
\end{aligned}
$$

Yield locus

$$
\mathcal{F}=J^{2}-\frac{M^{2}}{3} p^{\prime} \times\left(p_{0}^{*}-p^{\prime}\right)=0
$$
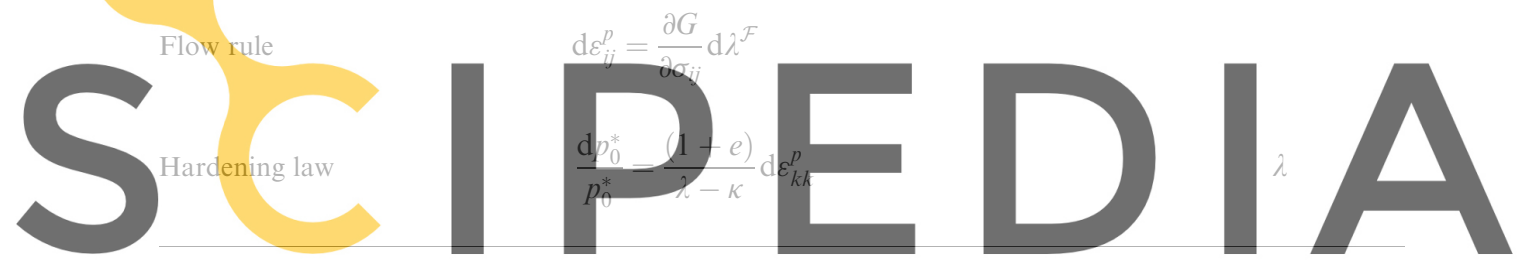

Register for free at https//www.scipedia.com to download the version without the watermark represented by a three-dimensional yield surface in the $p^{\prime \prime}-J-s$ diagram (see Fig. 1b). This surface is called loading-collapse (LC) yield surface. The elastic domain is closed by a second yield surface called suction increase (SI), coupled to the LC by the volumetric plastic strain. This model is able to reproduce the main features of the deformational response of an unsaturated soil:

- increase of the shear strength and preconsolidation pressure with suction, described by the dependency of $p_{0}$ and $p_{s}$ on suction;

- development of reversible swelling strains when suction decreases at low confining stress, that is in the elastic domain (path AB in Fig. 2);

- occurrence of irreversible collapse strains when suction decreases at high confining stress, that is activating the LC yield surface (path CD in Fig. 2);

- development of irreversible shrinkage when suction increases above a threshold value, that is activating the SI yield surface (path AE in Fig. 2).

When $s=0$ (saturated condition), modified Cam clay model is recovered.

The model works with 12 parameters, five of them being the same as in the modified Cam clay model ( $\kappa, G, M, p_{0}^{*}$ and $\lambda(0)$, the latter corresponding to $\lambda$ in MCCM). $p_{c}, r$ and $\theta$ define the change of the yield locus with suction and can be estimated when the LC 

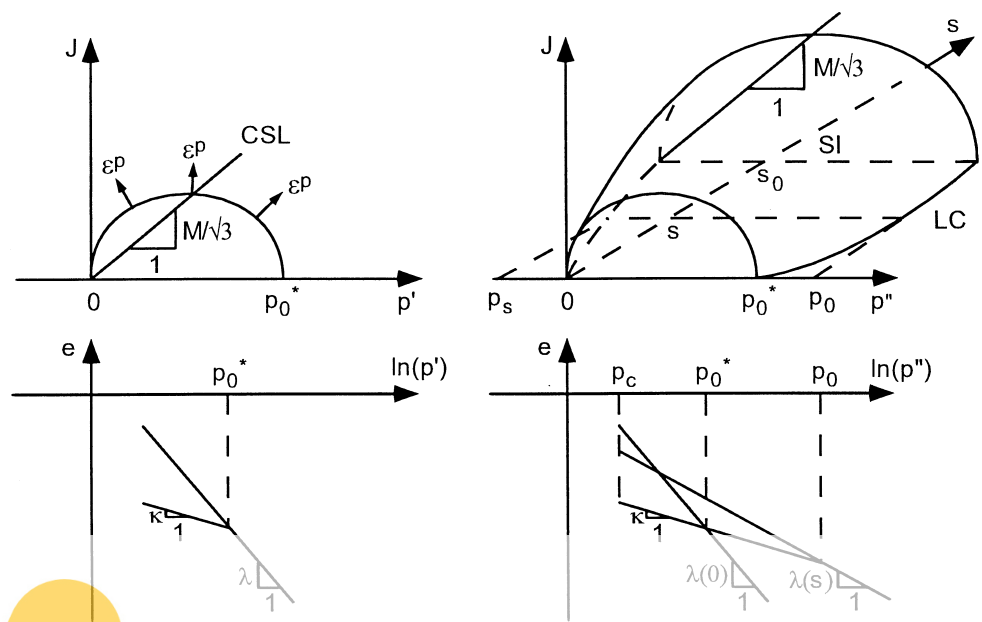

a) Modified Cam clay model

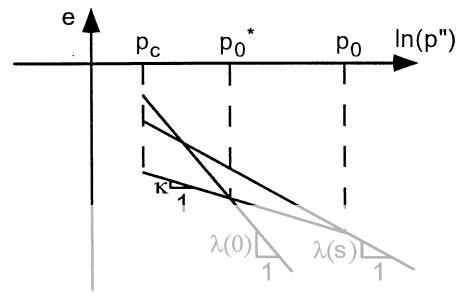

b) Extension to unsaturated conditions

Fig. 1. Cam clay model and BBM model

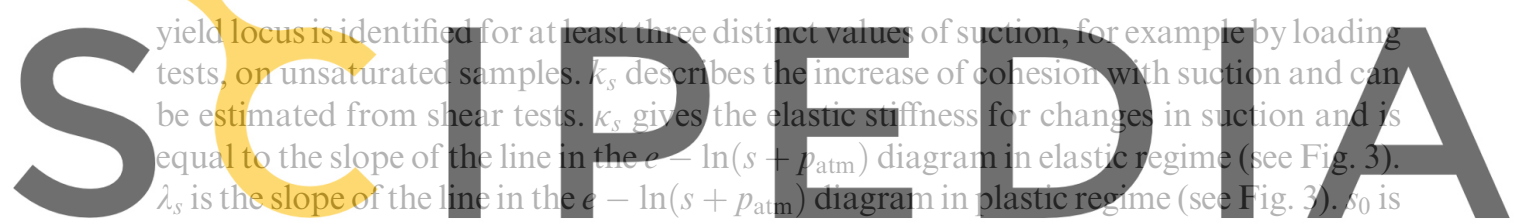

a history variable equal to the maximum suction known by the material. More details on

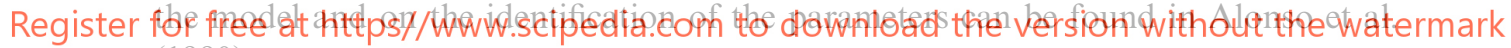
(1990).

\section{Generalised variables for unsaturated media}

For algorithmic convenience, the following generalised variables are defined. It is to note that they are not thermodynamically conjugated:

$$
\hat{\boldsymbol{\sigma}}={ }^{t}\left(\sigma_{11}^{\prime \prime}, \sigma_{22}^{\prime \prime}, \sigma_{33}^{\prime \prime}, \tau_{12}, \tau_{13}, \tau_{23}, s\right) \hat{\boldsymbol{\epsilon}}={ }^{t}\left(\epsilon_{11}^{\sigma}, \epsilon_{22}^{s}, \epsilon_{33}^{\sigma}, \epsilon_{12}^{\sigma}, \epsilon_{13}^{\sigma}, \epsilon_{23}^{\sigma}, \epsilon_{v}^{s}\right)
$$

where $\epsilon_{i j}^{\sigma}$ are strain components due to net stress changes at fixed suction and $\epsilon_{v}^{s}$ is the volumetric strain due to suction changes at fixed net stresses. Total strain component $\epsilon_{i j}$ can be deduced from $\epsilon_{i j}^{\sigma}$ and $\epsilon_{v}^{s}$ by:

$$
\epsilon_{i j}=\epsilon_{i j}^{\sigma}+\delta_{i j} \frac{\epsilon_{v}^{s}}{3}
$$

Equations of BBM in the space of generalised variables are described in Table 3 ( $\hat{p}$ denotes the value of $p^{\prime \prime}$ and $\hat{J}$ the value of $J$ in the new space). 
Table 2

Barcelona basic model

Elastic part

$$
\begin{aligned}
& \mathrm{d} \varepsilon_{i j}^{e}=\frac{2 G-3 K}{6 K G} \mathrm{~d} p_{i j}^{\prime \prime}+\frac{1}{2 G} \mathrm{~d} \sigma_{i j}^{\prime \prime}+\frac{1}{3 K^{s}} \mathrm{~d} s \delta_{i j} \\
& K=\frac{(1+e) p^{\prime \prime}}{\kappa}, G=\text { Cste, } K^{s}=\frac{(1+e)\left(s+p_{\mathrm{atm}}\right)}{\kappa_{s}} \quad k, G, \kappa_{s}
\end{aligned}
$$

Yield loci

$$
\mathcal{F}^{L C}=J^{2}-\frac{M^{2}}{3}\left(p^{\prime \prime}+p s\right)\left(p_{0}-p^{\prime \prime}\right)=0
$$

$$
\lambda(s)=\lambda(0)[r+(1-r) \exp (-\theta s)]
$$
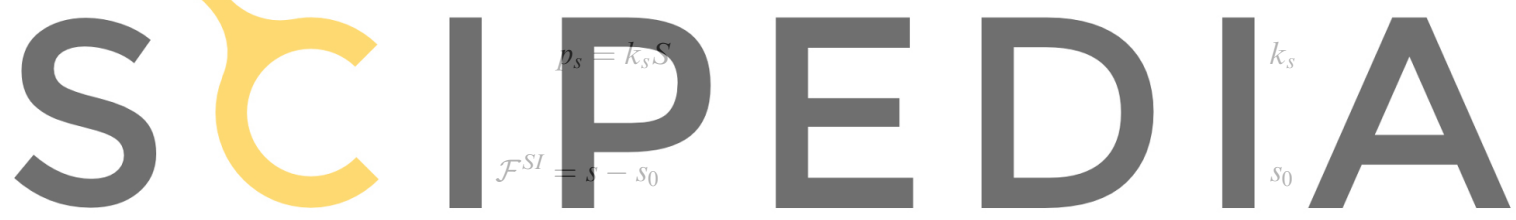

Register for free at https//www.scipedia.com to download the version without the watermark

Plastic potentials

$\mathcal{G}^{L C}=\alpha J^{2}-\frac{M}{3}\left(p^{\prime \prime}+p_{s}\right)\left(p_{0}-p^{\prime \prime}\right)$

$$
\begin{aligned}
& \text { with } \alpha=\frac{M(M-9)(M-3)}{9(6-M)} \frac{1}{1-\kappa / \lambda(0)} \\
& G^{S I}=p^{\prime \prime}
\end{aligned}
$$

Flow rule

$$
\mathrm{d} \varepsilon_{i j}^{p}=\frac{\partial \mathcal{G}^{\mathrm{LC}}}{\partial \sigma_{i j}^{\prime \prime}} \mathrm{d} \lambda^{\mathrm{LC}}+\frac{\partial \mathcal{G}^{\mathrm{SI}}}{\partial \sigma_{i j}^{\prime \prime}} \mathrm{d} \lambda^{\mathrm{SI}}
$$

Hardening laws

$$
\begin{aligned}
& \frac{\mathrm{d} p_{0}^{*}}{p_{0}}=\frac{(1+e)}{\lambda(0)-\kappa} \mathrm{d} \varepsilon_{k k}^{p} \\
& \frac{\mathrm{d} s_{0}}{s_{0}+p_{\mathrm{atm}}}=\frac{(1+e)}{\lambda_{s}-\kappa_{s}} \mathrm{~d} \varepsilon_{k k}^{p}
\end{aligned}
$$




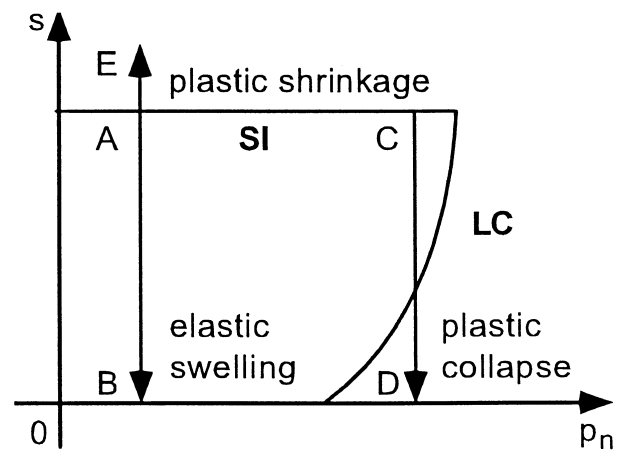

Fig. 2. Main predictive features of BBM.
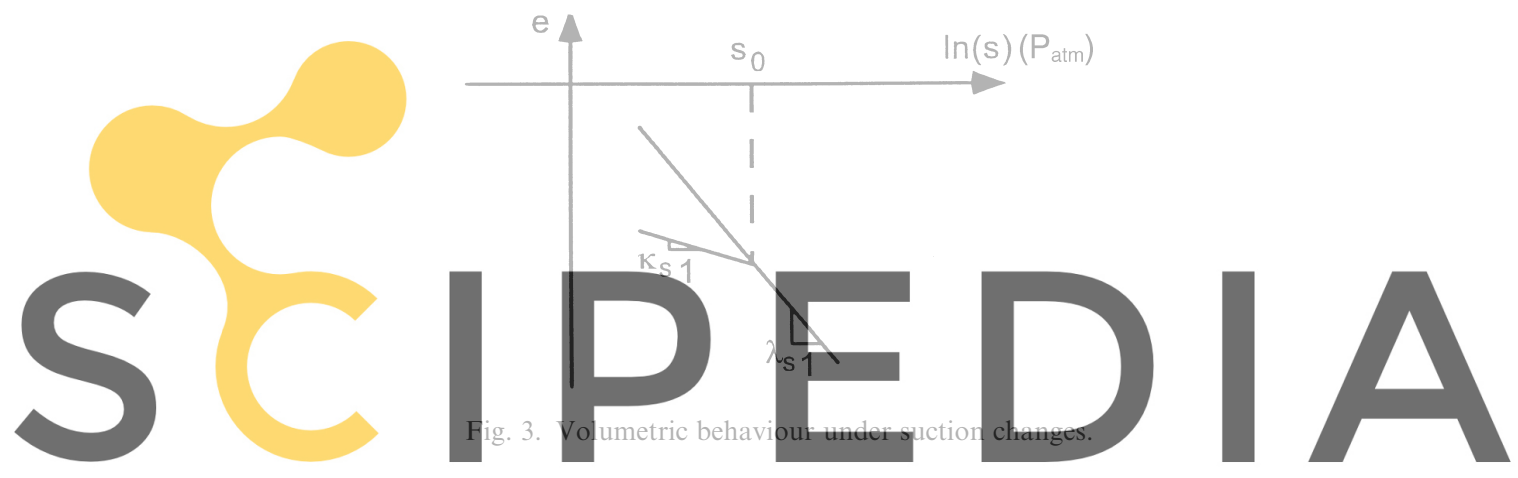

Register for free at https//www.scipedia.com to download the version without the watermark

Since the first $6 \times 6$ submatrix of the generalised elastic stiffness matrix $\hat{\mathbb{D}}$ is positive definite with the restrictions $K>0$ and $G>0, \hat{\mathbb{D}}$ is positive definite with the additional restriction $K^{s}>0$. Isovalues of $\hat{\mathcal{G}}^{\mathrm{LC}}$ describe elliptical cylinders in the generalised space $\hat{p}-\hat{J}-s$ and the LC plastic potential is non associated. On the other hand, SI plastic potential becomes associated in the new space.

\section{Numerical implementation}

\subsection{Strain driven discrete formulation}

\subsubsection{Elastic trial}

Bulk modulus $K$ is pressure dependent and the material has a nonlinear behaviour in the elastic domain. Various techniques can be used to handle such nonlinearities: implementation of a predictor-corrector scheme or use of secant elastic matrix as proposed by Borja (1991). This problem is not addressed in the present paper and elastic parameters are frozen at the beginning of the step $n+1$. Elastic trial reads then:

$$
\hat{\boldsymbol{\sigma}}_{n+1}=\hat{\boldsymbol{\sigma}}_{n}+\hat{\mathbf{D}}_{n} \Delta \hat{\boldsymbol{\epsilon}}_{n+1}
$$


Table 3

BBM model in the space of generalised variables

Elastic part

$$
\begin{aligned}
& \mathrm{d} \hat{\boldsymbol{\sigma}}=\hat{\mathbf{D}} \mathrm{d} \hat{\boldsymbol{\varepsilon}}^{e} \\
& \text { with } \hat{\mathbf{D}}=\left(\begin{array}{ccccccc}
K+\frac{4}{3} G & K-\frac{2}{3} G & K-\frac{2}{3} G & 0 & 0 & 0 & 0 \\
K-\frac{2}{3} G & K+\frac{4}{3} G & K-\frac{2}{3} G & 0 & 0 & 0 & 0 \\
K-\frac{2}{3} G & J-\frac{2}{3} G & K+\frac{4}{3} G & 0 & 0 & 0 & 0 \\
0 & 0 & 0 & 2 G & 0 & 0 & 0 \\
0 & 0 & 0 & 0 & 2 G & 0 & 0 \\
0 & 0 & 0 & 0 & 0 & 2 G & 0 \\
0 & 0 & 0 & 0 & 0 & 0 & K_{s}
\end{array}\right)
\end{aligned}
$$

Yield loci

$$
\hat{\mathcal{F}}^{\mathrm{LC}}=\hat{J}^{2}-\frac{M^{2}}{3}\left(\hat{p}+p_{s}\right)\left(p_{0}-\hat{p}\right)=0
$$

$$
\begin{aligned}
& \frac{p_{0}}{p c}=\left(\frac{p_{0}^{*}}{p c}\right)^{(\lambda(0)-\kappa) /(\lambda(s)-\kappa)} \\
& \lambda\left(\widehat{\sigma}_{7}\right)=\lambda(0)\left[r+(1-r) \exp \left(-\theta \widehat{\sigma_{7}}\right)\right]
\end{aligned}
$$
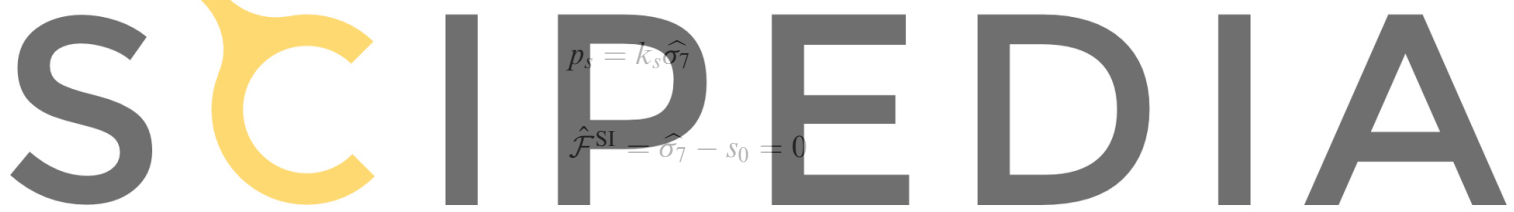

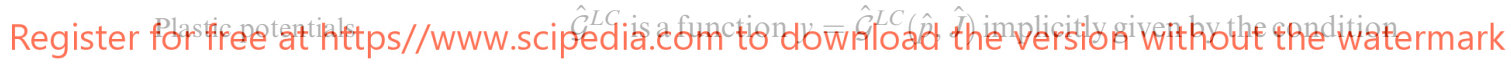

$$
\begin{aligned}
& \alpha \hat{J}^{2}-\frac{M^{2}}{3}\left(\hat{p}+p_{s}(y)\right)\left(p_{0}(y)-\hat{p}\right)=0^{a} \\
& \text { with } \alpha=\frac{M(M-9)(M-3)}{9(6-M)} \frac{1}{1-\kappa / \lambda(0)} \\
& \hat{G}^{S I}=\widehat{\sigma}_{7}-s_{0} \\
& \mathrm{~d} \hat{\varepsilon}^{p}=\frac{\partial \hat{G}^{\mathrm{LC}}}{\partial \hat{\sigma}} \mathrm{d} \lambda^{\mathrm{LC}}+\frac{\partial \hat{G}^{\mathrm{SI}}}{\partial \hat{\sigma}} \mathrm{d} \lambda^{\mathrm{SI}} \\
& \frac{\mathrm{d} p_{0}^{*}}{p_{0}^{*}}=\frac{(1+e)}{(\lambda(0)-\kappa)}\left(\mathrm{d} \hat{\varepsilon}_{1}^{p}+\mathrm{d} \hat{\varepsilon}_{2}^{p}+\mathrm{d} \hat{\varepsilon}_{3}^{p}+\mathrm{d} \hat{\varepsilon}_{7}^{p}\right) \\
& \frac{\mathrm{d}_{S_{0}}}{s_{0}+p_{\text {atm }}}=\frac{(1+e)}{\left(\lambda_{s}-\kappa_{s}\right)}\left(\mathrm{d} \hat{\varepsilon}_{1}^{p}+\mathrm{d} \varepsilon_{2}^{p}+d \hat{\varepsilon}_{3}^{p}+d \hat{\varepsilon}_{7}^{p}\right)
\end{aligned}
$$

Flow rules

Hardening laws

a It can be readily shown that $\frac{\partial \hat{G}^{\mathrm{LC}}}{\partial \mathrm{P}} / \frac{\partial \hat{G}^{\mathrm{LC}}}{\partial J}=\frac{\mathcal{G}^{\mathrm{LC}}}{\partial \mathrm{P}^{\prime \prime}} / \frac{\partial G^{\mathrm{LC}}}{\partial J}$ and $\frac{\partial \hat{G}^{\mathrm{LC}}}{\partial \widehat{\sigma}_{7}}=0$ 
Following the procedure developed by Simo et al. (1988), active yield surfaces will be searched within the set of admissible yield surfaces defined by $\hat{\mathcal{F}}^{\beta}\left(\hat{\boldsymbol{\sigma}}_{n+1}, \hat{\boldsymbol{X}}_{n}\right) \geqslant 0, \beta=$ LC, SI where $\hat{\chi}_{n}$ is the vector of hardening variables obtained at the end of the previous step. This is not necessarily true on the softening side of the critical states. As a consequence, the following algorithm, although general when a unique yield surface is active, is only valid for the hardening zone (right side of critical states in the $p^{\prime \prime}-J-s$ diagram) in the case of simultaneous activation of LC and SI.

\subsubsection{Return mapping}

Correction to yield surface is performed in order to respect the discrete evolution equations over a time step $\Delta t$. Simo et al. (1988) have presented a comprehensive formulation of these equations for the case of isotropic and kinematic hardening plasticity and visco-plasticity, using a backward Euler time discretisation. This formulation can be extended to the generalised variables defined in Section 3 and noting:

$$
\hat{\mathbf{F}}={ }^{t}\left(\hat{\mathcal{F}}^{L C}, \hat{\mathcal{F}}^{S I}\right), \hat{\mathbf{G}}=\left(\hat{\mathcal{G}}^{L C}, \hat{\mathcal{G}}^{S I}\right), \hat{\boldsymbol{\chi}}={ }^{t}\left(p_{0}^{*}, s_{0}\right) \text { and } \hat{\boldsymbol{\lambda}}={ }^{t}\left(\hat{\lambda}^{L C}, \hat{\lambda}^{S I}\right)
$$

the system to solve in the case of isotropic hardening plasticity becomes:

$$
\begin{aligned}
& \hat{\boldsymbol{\epsilon}}_{n+1}=\hat{\boldsymbol{\epsilon}}_{n}+\Delta \hat{\boldsymbol{\epsilon}}_{n+1} \\
& \hat{\boldsymbol{\sigma}}_{n+1}=\hat{\boldsymbol{\sigma}}_{n}+\Delta \hat{\boldsymbol{\sigma}}_{n+1} \\
& \hat{\boldsymbol{\chi}}_{n+1}=\hat{\boldsymbol{\chi}}_{n}+\Delta \hat{\boldsymbol{\chi}}_{n+1}
\end{aligned}
$$

with

$$
\begin{aligned}
\Delta \hat{\boldsymbol{\epsilon}}_{n+1}^{p} & =\left.\frac{\partial \hat{\mathbf{G}}}{\partial \hat{\boldsymbol{\sigma}}}\right|_{n+1} \Delta \hat{\boldsymbol{\lambda}}_{n+1} \\
\Delta \hat{\boldsymbol{\sigma}}_{n+1} & =\hat{\mathbf{D}}\left(\Delta \hat{\boldsymbol{\epsilon}}_{n+1}-\Delta \hat{\boldsymbol{\epsilon}}_{n+1}^{p}\right) \\
\Delta \hat{\boldsymbol{\chi}}_{n+1} & =\left.\frac{\partial \hat{\boldsymbol{\chi}}}{\partial \hat{\boldsymbol{\epsilon}}^{p}}\right|_{n+1} \Delta \hat{\boldsymbol{\epsilon}}_{n+1}^{p}
\end{aligned}
$$

where superscript $p$ means plastic. $\Delta \hat{\boldsymbol{\lambda}}_{n+1}, \Delta \hat{\boldsymbol{\sigma}}_{n+1}$ and $\Delta \hat{\boldsymbol{\chi}}_{n+1}$ are constrained by the discrete form of Kuhn Tucker conditions:

$$
\Delta \hat{\lambda}_{n+1}^{\beta} \hat{\mathcal{F}}_{n+1}^{\beta}=0 \text { with } \Delta \hat{\lambda}_{n+1}^{\beta} \geqslant 0 \text { and } \hat{\mathcal{F}}_{n+1}^{\beta} \leqslant 0 \quad \beta=\text { LC, SI }
$$

Substitution of Eq. (6) into Eqs. (7)-(9) leads to a system of non linear equations having as unknowns $\Delta \hat{\boldsymbol{\sigma}}_{n+1}, \Delta \hat{\boldsymbol{\chi}}_{n+1}$ and $\Delta \hat{\boldsymbol{\lambda}}_{n+1}$. To solve it, the Newton procedure described in the Appendix is used.

\subsection{Mixed control}

As described in the introduction, increment of total strain $\Delta \epsilon_{i j}$ and increment of suction $\Delta s$ are generally given as input of the elastoplastic subroutine in most FE formulations. Required output is the increment of net stress vector over the prescribed discrete time step. With the generalised variables previously defined, a 
mixed stress-strain controlled procedure has to be implemented to perform the integration. This mixed control is of two types:

1. the strain increment components $\Delta \epsilon_{i j}^{\sigma}$ are not imposed but constrained by the input value of the total strain component

$$
\Delta \epsilon_{i j}=\Delta \epsilon_{i j}^{\sigma}+\delta_{i j} \Delta \epsilon_{v}^{s}
$$

2. the strain component $\Delta \epsilon_{v}^{s}$ is not given but the elastic part $\Delta \epsilon_{v}^{s e}$ is fixed by the prescribed increment of suction $\Delta s$ and the plastic part $\Delta \epsilon_{v}^{s p}$ evolves according to the hardening laws of the yield surfaces.

Mixed control in the classical stress-strain space was studied by Alawaji et al. $(1990,1992)$ for the cases of drained and undrained responses. Two main techniques were presented by these authors. The first one consists in using a strain driven core within iterative corrections to reach stress driven conditions. This technique is typically used when integration of the constitutive law is done by an implicit scheme. The second one consists in partially inverting the stress-strain relationship for the prescribed degrees of freedom and is generally contemplated for explicit stress point algorithms. A hybrid method will be presented here, which consists in computing the elastic trial by partial inversion of the stiffness matrix and in keeping the strain driven structure during elastoplastic corrections.

The following strain vectors are defined:

$$
\begin{aligned}
& \hat{\boldsymbol{\epsilon}}^{*}={ }^{t}\left(\hat{\epsilon}_{1}+\frac{1}{3} \hat{\epsilon}_{7}, \hat{\epsilon}_{2}+\frac{1}{3} \hat{\epsilon}_{7}, \hat{\epsilon}_{3}+\frac{1}{3} \hat{\epsilon}_{7}, \hat{\epsilon}_{4}, \hat{\epsilon}_{5}, \hat{\epsilon}_{6}, \hat{\epsilon}_{7}\right) \\
& \hat{\boldsymbol{\epsilon}}^{e *}={ }^{t}\left(\hat{\epsilon}_{1}^{e}+\frac{1}{3} \hat{\epsilon}_{7}^{e}, \quad \hat{\epsilon}_{2}^{e}+\frac{1}{3} \hat{\epsilon}_{7}^{e}, \quad \hat{\epsilon}_{3}^{e}+\frac{1}{3} \hat{\epsilon}_{7}^{e}, \quad \hat{\epsilon}_{4}^{e}, \hat{\epsilon}_{5}^{e}, \hat{\epsilon}_{6}^{e}, \hat{\epsilon}_{7}^{e}\right) \\
& \hat{\boldsymbol{\epsilon}}^{p *}={ }^{t}\left(\hat{\epsilon}_{1}^{p}+\frac{1}{3} \hat{\epsilon}_{7}^{p}, \quad \hat{\epsilon}_{2}^{p}+\frac{1}{3} \hat{\epsilon}_{7}^{p}, \quad \hat{\epsilon}_{3}^{p}+\frac{1}{3} \hat{\epsilon}_{7}^{p}, \quad \hat{\epsilon}_{4}^{p}, \hat{\epsilon}_{5}^{p}, \hat{\epsilon}_{6}^{p}, \hat{\epsilon}_{7}^{p}\right)
\end{aligned}
$$

The six first components of $\hat{\boldsymbol{\epsilon}}^{*}$ are total strain components. Their increment is fixed at the beginning of the integration. The seventh component is stress-driven, in the sense that its elastic part is fixed by the increment of suction given as input.

Defining the elastic stiffness matrix by:

$$
\hat{\mathbf{D}}^{*}=\left(\begin{array}{ccccccc}
K+\frac{4}{3} G & K-\frac{2}{3} G & K-\frac{2}{3} G & 0 & 0 & 0 & -K \\
K-\frac{2}{3} G & K+\frac{4}{3} G & K-\frac{2}{3} G & 0 & 0 & 0 & -K \\
K-\frac{2}{3} G & K-\frac{2}{3} G & K+\frac{4}{3} G & 0 & 0 & 0 & -K \\
0 & 0 & 0 & 2 G & 0 & 0 & 0 \\
0 & 0 & 0 & 0 & 2 G & 0 & 0 \\
0 & 0 & 0 & 0 & 0 & 2 G & 0 \\
0 & 0 & 0 & 0 & 0 & 0 & K^{s}
\end{array}\right)
$$


the elastic trial is equal to $\hat{\mathbf{D}}^{*} \Delta \hat{\boldsymbol{\epsilon}}^{*}$ where $\Delta \hat{\epsilon}_{7}^{*}$ is set to $\Delta s / K^{s}$. The flow rule becomes $\mathrm{d} \hat{\boldsymbol{\epsilon}}^{p^{*}}=\partial \hat{\mathbf{G}}^{*} / \partial \hat{\boldsymbol{\sigma}} \mathrm{d} \hat{\boldsymbol{\lambda}}$ with:

$$
\frac{\partial \hat{G}^{\beta^{*}}}{\partial \hat{\boldsymbol{\sigma}}}={ }^{t}\left(\frac{\partial \mathcal{G}^{\beta}}{\partial \hat{\sigma}_{1}}+\frac{1}{3} \frac{\partial \mathcal{G}^{\beta}}{\partial \hat{\sigma}_{7}}, \frac{\partial \mathcal{G}^{\beta}}{\partial \hat{\sigma}_{2}}+\frac{1}{3} \frac{\partial \mathcal{G}^{\beta}}{\partial \hat{\sigma}_{7}}, \frac{\partial \mathcal{G}^{\beta}}{\partial \hat{\sigma}_{3}}+\frac{1}{3} \frac{\partial \mathcal{G}^{\beta}}{\partial \hat{\sigma}_{7}}, \frac{\partial \mathcal{G}^{\beta}}{\partial \hat{\sigma}_{4}}, \frac{\partial \mathcal{G}^{\beta}}{\partial \hat{\sigma}_{5}}, \frac{\partial \mathcal{G}^{\beta}}{\partial \hat{\sigma}_{6}}, \frac{\partial \mathcal{G}^{\beta}}{\partial \hat{\sigma}_{7}}\right) \beta=\mathrm{LC}, \mathrm{SI}
$$

and the residual of generalised stresses during elastoplastic corrections reads:

$$
\mathbf{r}_{n+1}^{\sigma i+1}=\Delta \hat{\boldsymbol{\sigma}}_{n+1}^{i+1}-\hat{\mathbf{D}}_{n}^{*}\left(\Delta \hat{\boldsymbol{\epsilon}}_{n+1}^{*}-\left.\frac{\partial \hat{\mathbf{G}}^{*}}{\partial \hat{\boldsymbol{\sigma}}}\right|_{n+1} ^{i+1} \Delta \hat{\boldsymbol{\lambda}}_{n+1}^{i+1}\right)
$$

$\Delta \hat{\boldsymbol{\epsilon}}_{n+1}^{*}$ is not fixed any more during the return mapping corrections. Derivatives of the residual $\mathbf{r}^{\sigma}$ with respect to the generalised stresses take the form [see Eq. (A6) in the Appendix]:

$$
\frac{\partial \mathbf{r}^{\sigma}}{\partial \hat{\boldsymbol{\sigma}}}=\mathbf{I}-\hat{\mathbf{D}}^{*} \frac{\partial \hat{\boldsymbol{\epsilon}}^{*}}{\partial \hat{\boldsymbol{\sigma}}}+\sum_{\beta=\mathrm{LC}, \mathrm{SI}} \Delta \hat{\lambda}^{\beta} \hat{\mathbf{D}}^{*} \frac{\partial^{2} \hat{\mathcal{G}}^{\beta}}{\partial \hat{\boldsymbol{\sigma}}^{2}}
$$

$\frac{\partial \hat{\boldsymbol{\epsilon}}^{*}}{\partial \hat{\boldsymbol{\sigma}}}$ can be split into elastic and plastic parts:

$$
\frac{\partial \hat{\boldsymbol{\epsilon}}^{*}}{\partial \hat{\boldsymbol{\sigma}}}=\frac{\partial \hat{\boldsymbol{\epsilon}}^{\mathrm{e}}}{\partial \hat{\boldsymbol{\sigma}}}+\frac{\partial \hat{\boldsymbol{\epsilon}}^{p *}}{\partial \hat{\boldsymbol{\sigma}}}=\frac{\partial \hat{\boldsymbol{\epsilon}}^{\mathrm{e} *}}{\partial \hat{\boldsymbol{\sigma}}}\left(\mathbf{I}+\frac{\partial \hat{\boldsymbol{\epsilon}}^{p *}}{\partial \hat{\boldsymbol{\epsilon}}^{\mathrm{e} *}}\right)=\hat{\mathbf{D}}^{*-1}\left(\mathbf{I}+\frac{\partial \hat{\boldsymbol{\epsilon}}^{p *}}{\partial \hat{\boldsymbol{\epsilon}}^{\mathrm{e} *}}\right)
$$

Defining $\mathrm{MC}=\partial \hat{\boldsymbol{\epsilon}}^{p *} / \partial \hat{\boldsymbol{\epsilon}}^{\mathrm{e} *}$, Eq. (15) reads:

$$
\frac{\partial \mathbf{r}^{\sigma}}{\partial \hat{\boldsymbol{\sigma}}}=-M C+\sum_{\beta=\mathrm{LC}, \mathrm{SI}} \Delta \hat{\lambda}^{\beta} \hat{\mathbf{D}}^{*} \frac{\partial^{2} \hat{\mathcal{G}}^{\beta^{*}}}{\partial \hat{\boldsymbol{\sigma}}^{2}}
$$

MC is a diagonal matrix. For the strain driven subscripts $(i=1-6), \mathrm{d} \hat{\epsilon}_{i}^{\mathrm{e} *}=-\mathrm{d} \hat{\epsilon}_{i}^{p *}$ and the term on the diagonal is equal to -1 . For the stress driven subscripts $(i=7)$, $\mathrm{d} \hat{\epsilon}_{i}^{\mathrm{e} *}=0$ and the term is infinite.

In the numerical implementation of the global Jacobian matrix, infinite terms of MC are expressed by a very high value. These coefficients act as penalisers which impose $\delta \hat{\boldsymbol{\sigma}}_{n+1}=\mathbf{0}$ for the stress driven subscripts. In this way, no additional iteration loop is needed to fulfil the condition imposed on the values of the fixed stress components.

\section{Analysis of results}

Proposed implementation is validated over various generalised stress-generalised strain paths. Four basic tests are run. In test A, activation of SI by drying is checked. In tests B and C, activation of LC by loading and wetting is verified. In test 
D, two scenarios of corner activation are studied (tests D1 and D2). The material considered is a silt of low plasticity $\left(w_{\mathrm{L}}=37 \%, w_{\mathrm{P}}=18-21 \%\right)$ originating from Jossigny - France. Parameters of the material are given in Table 4. Numerical outputs of tests A and B are compared with experimental results presented by Vicol (1990). No experimental results are available for tests C and D.

Test A corresponds to test labelled E3 in the work of Vicol (1990). Stress path is shown in Fig. 4a. Initially, the clay paste is saturated and not loaded. $p_{0}^{*}$ and $s_{0}$ are then equal to 0 . As a result of the vertical loading up to $25 \mathrm{kPa}$, hardening takes place for both LC and SI and $s_{0}$ takes the value of $16.4 \mathrm{kPa}$ at point A. During drying, strains are, therefore, elastic up to a value of $16.4 \mathrm{kPa}$ (path $\mathrm{A}-\mathrm{B}$ in Fig. 4b). Afterwards, elastoplastic strains develop (path B-C). When a suction of $100 \mathrm{kPa}$ is reached, a wetting/redrying path in the elastic domain is imposed (path C-D-C) followed by a new increase of suction up to a value of $1500 \mathrm{kPa}$ (path $\mathrm{C}-\mathrm{E}$ ) in the elastoplastic regime. Numerical results can be favourably compared with laboratory data. However, at suction higher than $400 \mathrm{kPa}$, measured strain increment appears to be smaller than the computed one, that could indicate a nonlinear dependency of $\lambda_{s}$ with suction in the reality. Test A validates activation of SI.

Test B corresponds to test E15 performed by Vicol (1990). As shown in Fig. 5a, the sample is first loaded in oedometer saturated conditions $\left(s=0, \epsilon_{2}=\epsilon_{3}=0\right)$ up to vertical stress of $25 \mathrm{kPa}$ (point A) and then dried up to a suction of $200 \mathrm{kPa}$ (point B). At that point, suction is maintained constant and the load is increased again up to $1600 \mathrm{kPa}$ (point D). Finally, the sample is unloaded at fixed suction up to $100 \mathrm{kPa}$ (point E). In the computation, loading paths are simulated by imposing a vertical strain until the prescribed vertical stress is reached. Evolution of void ratio vs logarithm of vertical stress is depicted in Fig. 5b. In saturated condition (path $\mathrm{O}-\mathrm{A}$ ),

Table 4

Properties for Jossigny's silt

\begin{tabular}{lllllllllll}
\hline Parameter & $\kappa$ & $\lambda$ & $\kappa_{s}$ & $\lambda_{s}$ & $p_{c}(\mathrm{kPa})$ & $r$ & $\theta\left(\mathrm{kPa}^{-1}\right)$ & $\nu^{\mathrm{a}}$ & $M$ & $k_{s}$ \\
\hline Value & 0.015 & 0.108 & 0.0012 & 0.032 & $6.54710^{-3}$ & 0.911 & $5.7510^{-3}$ & 0.3 & 1 & 0.6
\end{tabular}

a A constant Poisson's ratio $v$ has been used in spite of a constant $G$ for this material.
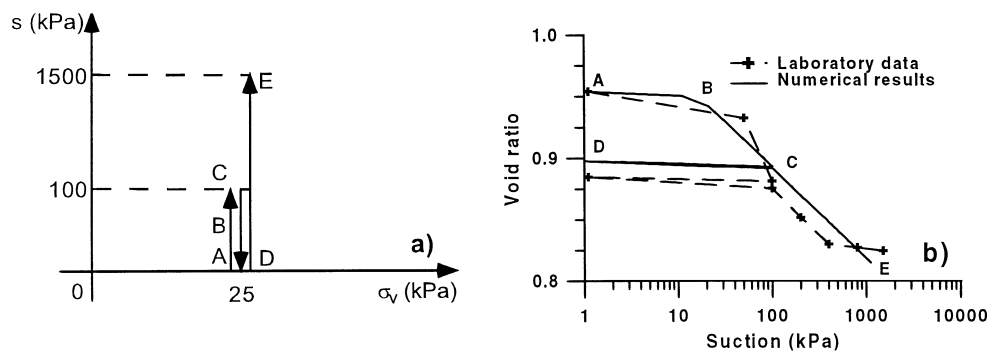

Fig. 4. Test A: (a) prescribed input; (b) detormational response. 
the oedometer virgin loading line is followed. During this stage, LC and SI harden and value of $p_{0}^{*}$ and $s_{0}$ at point $\mathrm{A}$ are, respectively, 22.24 and $16.47 \mathrm{kPa}$. Path $\mathrm{A}-\mathrm{B}$ is similar to path A-C in test A. During this path, activation of SI produces a shift of LC. During the subsequent loading, vertical strain is then elastic up to point $\mathrm{C}$ and elastoplastic between $\mathrm{C}$ and $\mathrm{D}$. The slope of the $e-\ln (s)$ line between $\mathrm{C}$ and $\mathrm{D}$ is slightly lower than between $\mathrm{O}$ and $\mathrm{A}$, because of the decrease of $\lambda(s)$ when suction increases. Paths $0-\mathrm{A}$ and $\mathrm{B}-\mathrm{D}-\mathrm{E}$ validate the activation of $\mathrm{LC}$ by applying deformation, that is the strain driven component of the generalised variable.

In test $\mathrm{C}$, a fictitious swelling pressure test at low (test $\mathrm{C}$, path $\mathrm{A}-\mathrm{B}-\mathrm{C}$ in Fig. 6) and high (test $\mathrm{C} 2$, path $\mathrm{D}-\mathrm{E}-\mathrm{F}-\mathrm{E}-\mathrm{G}$ in Fig. 6) confining stress is simulated. Initially, a suction equal to $1500 \mathrm{kPa}$ and two isotropic stress states, respectively equal to 42 and $95 \mathrm{kPa}$, are considered. Hydration is then simulated in order to reduce suction up to a null value at constant total volume $\left(\epsilon_{v}=0\right)$. Since the total volumetric strain is equal to $0, \mathrm{LC}$ can experiment only a small hardening to compensate the volumetric elastic strain. Moreover, because the generalised stress path is always directed towards the region of unreachable stress state, it should follow the LC yield surface. This type of test provides thus a clear check of LC activation under mixed control. As depicted in Fig. 6, at low confining stress, most of the hydration process takes
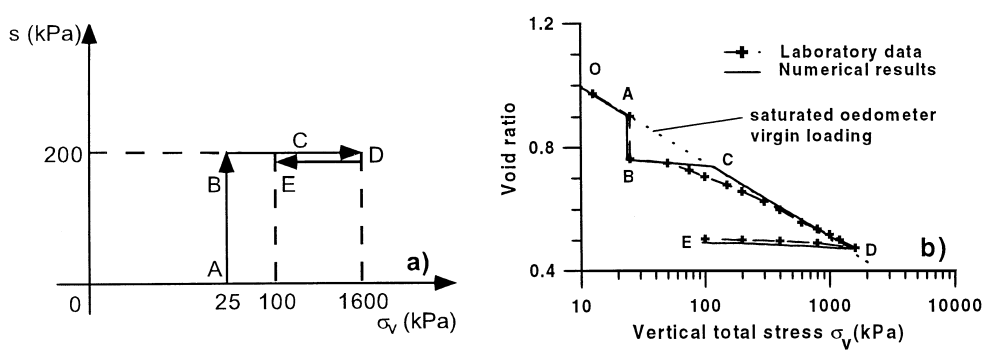

Fig. 5. Test B: (a) prescribed input; (b) deformational response.

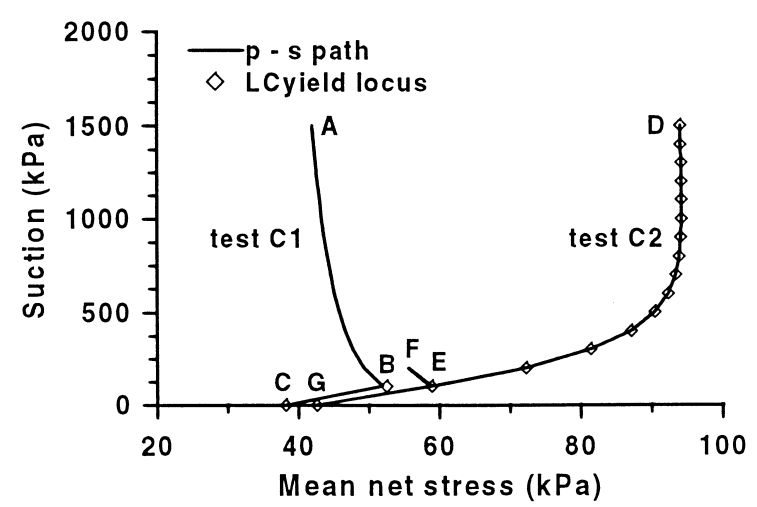

Fig. 6. Test C: Stress path in the mean net stress-suction diagram. 
place in the elastic zone (path A-B). At high confining stress, LC is reached at the beginning of the test and elastoplastic regime is activated (path D-G). For both paths, computed mean net stress $p^{\prime \prime}$ is equal to the hardening parameter $p_{0}$ when LC is reached (paths $\mathrm{B}-\mathrm{C}$ and $\mathrm{D}-\mathrm{E}-\mathrm{G}$ ). A small hardening of $\mathrm{LC}$ can be observed between tests $\mathrm{Cl}$ and $\mathrm{C} 2$, because a sooner activation of $\mathrm{LC}$ produces higher amount of plastic strains to compensate elastic strains at constant volume. For test $\mathrm{C} 2$, a drying/rewetting path (path $\mathrm{E}-\mathrm{F}-\mathrm{E}$ ) has also been imposed to check the loading/ unloading condition in the generalised stress space.

Test D aims at checking corner activation. In test D1, parameters of Jossigny's silt are used. Initially, suction is equal to $500 \mathrm{kPa}$ and the mean net stresses to $25 \mathrm{kPa}$. Values for $p_{0}^{*}$ and $s_{0}$ are, respectively, 35 and $520 \mathrm{kPa}$. A total volumetric deformation of $6 \%$ and an increase of suction of $200 \mathrm{kPa}$ is imposed. As can be seen in Fig. 7a, SI is activated at point A, accompanied by hardening of LC. At point B, corner is reached and since hardening of LC is lower than hardening of SI, the loading-drying path remains henceforth on LC yield surface (path B-C). In test D2, value of parameter $\lambda_{s}$ is changed to 0.4 and initial value of $s_{0}$ fixed to $550 \mathrm{kPa}$. With this configuration LC has a quicker hardening than SI and is first activated. Fig. $7 \mathrm{~b}$ shows the resulting net stress-suction path. At point $\mathrm{A}, \mathrm{LC}$ is activated and corner is reached at point B. Subsequently, corner remains activated and an inflexion can be observed in the net stress-suction path imposed by the hardening law of SI. Relevance of dealing with corner activation appears in this example: when LC hardens more rapidly than SI, any path activating LC and directed towards SI will lead to a permanent corner activation, if sufficiently maintained.

Table 5 summarises some performances of the algorithm for the distinct tests. Convergence of Newton-Raphson procedure is defined by two simultaneous criteria: (1) the Euclidean norm of the residual vector is less than a given tolerance; (2) the Euclidean norm of the unknown correction vector is less than a given tolerance. In all the tests $\mathrm{A}, \mathrm{B}, \mathrm{C}$ and $\mathrm{D}$, both tolerances are fixed to $10^{-8}$, considering $\mathrm{kPa}$ as stress unit. Mean number of iterations per plastic step (step when at least one yield surface is active) is typically below 7 for reasonably small load increment. For the present set of parameters, LC activation by strain increment (test B) appears to have the most critical convergence rate. As shown in Fig. 8a, convergence rate decreases with the level of stresses as a result of the logarithmic variation of the plastic parameters, and may not be reached for some loading patterns (case of 23 load steps).
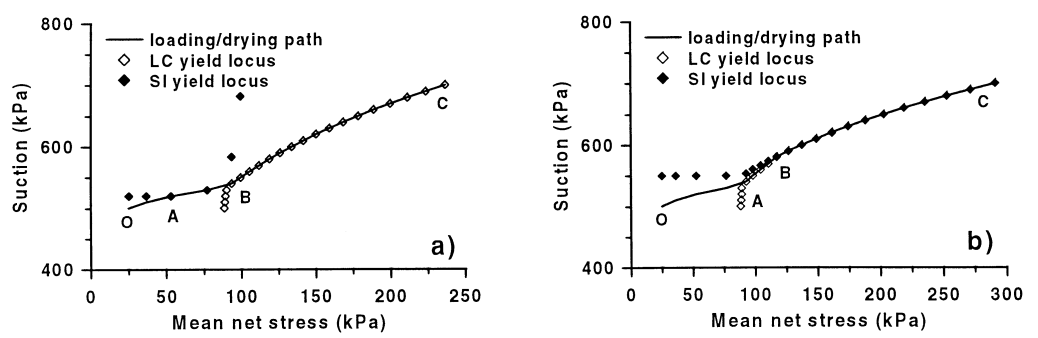

Fig. 7. Test D: (a) Corner activation for case D1; (b) corner activation for case D2. 
Table 5

test performance

\begin{tabular}{lrll}
\hline Test & Number of steps & $\begin{array}{l}\text { Prescribed } \\
\text { input in each step }\end{array}$ & $\begin{array}{l}\text { Mean number of iterations } \\
\text { per plastic step }\end{array}$ \\
\hline A & 150 & $\Delta s=10 \mathrm{kPa}$ & 4.20 \\
path DE & 15 & $\Delta s=100 \mathrm{kPa}$ & 5.58 \\
& 2 & $\Delta s=1000 \mathrm{kPa} \& \Delta s=500 \mathrm{kPa}$ & 7.00 \\
B paths & 230 & $\varepsilon_{1}=0.001$ & 5.26 \\
OA \& CD & 46 & $\varepsilon_{1}=0.005$ & 6.61 \\
& 23 & $\varepsilon_{1}=0.01$ & do not coverge for $\sigma_{v}>1000 \mathrm{kPa}$ \\
C2 & 150 & $\Delta s=-10 \mathrm{kPa}$ & 3.26 \\
path DG & 15 & $\Delta s=-100 \mathrm{kPa}$ & 4.33 \\
& 2 & $\Delta s=-1000 \mathrm{kPa} \& \Delta s=-500 \mathrm{kPa}$ & 6.00 \\
D1 & 20 & $\Delta \varepsilon_{v}=0.003, \Delta s=10 \mathrm{kPa}$ & 6.30 \\
path OC & 4 & $\Delta \varepsilon_{v}=0.015, \Delta s=50 \mathrm{kPa}$ & 9.50 \\
& 2 & $\Delta \varepsilon_{v}=0.03, \Delta s=100 \mathrm{kPa}$ & 12.00 \\
D2 & 20 & $\Delta \varepsilon_{v}=0.003, \Delta s=10 \mathrm{kPa}$ & 5.00 \\
path OC & 4 & $\Delta \varepsilon_{v}=0.015, \Delta s=50 \mathrm{kPa}$ & 6.75 \\
& 2 & $\Delta \varepsilon_{v}=0.03, \Delta s=100 \mathrm{kPa}$ & 8.5 \\
\hline
\end{tabular}
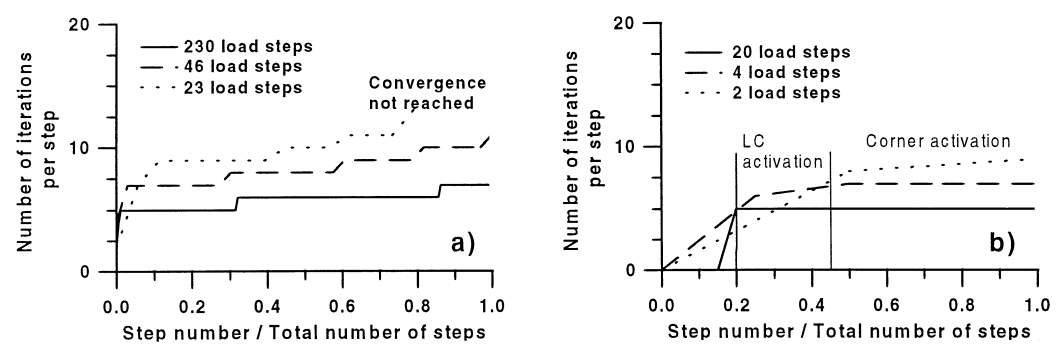

Fig. 8. (a) Convergence rate for path $\mathrm{CD}$ of test $\mathrm{B}$; (b) convergence rate for path $\mathrm{OA}$ of test $\mathrm{D} 2$.

Adaptative time stepping can be contemplated to deal with that problem. As concerns corner activation, the results depicted in Fig. $8 \mathrm{~b}$ indicate that no differences in terms of convergence rate exist with respect to one surface activation.

\section{Conclusions}

Integration of elastoplastic evolution equations within the framework of a FE formulation has been presented for the model of Alonso et al. (1990). This model has two peculiar features from the point of view of the stress point algorithm: (1) suction is considered as an additional stress component; (2) the elastic domain in the net stress-suction space is bordered by two yield surfaces with a non smooth intersection. To deal with point (1), generalised stress and strain vectors have been defined. This artifice enabled recoping with point (2) by extending the implicit integration 
procedure presented by Simo et al. (1988) for non smooth multisurface plasticity. Finally, a mixed control was presented in order to adapt the previous strain driven procedure to the case when total strain increment and suction increment are given as input of the subroutine, as is the case in most of present FE codes. This procedure combines partial inversion of the elastic stiffness matrix with penalisation technique of the discrete elastoplastic equations and preserves a one loop iterative algorithm. Validation results show a good behaviour of the algorithm. According to the work of Simo et al. (1988), activation of one and multiple yield surfaces during hardening is comprehensively dealt with by the proposed procedure. The developed implementation can be extended to models having the same basic characteristics of the model of Alonso et al. (1990).

\section{Acknowledgements}

The support of the D.G.I.C.Y.T. through research grant PB95-0771 is gratefully acknowledged. The authors thank J. Alcoverro for fruitful discussions.

\section{Appendix. Solution procedure for return mapping equations}

Noting $\mathbf{r}_{n+1}^{\sigma i+1}$ and $\mathbf{r}_{n+1}^{x i+1}$ the respective residuals on stresses and hardening parameters at iteration $i+1$ and increment $n+1$, we have:

$$
\begin{aligned}
& \mathbf{r}_{n+1}^{\sigma i+1}=\Delta \hat{\boldsymbol{\sigma}}_{n+1}^{i+1}-\hat{\mathbf{D}}_{n}\left(\Delta \hat{\boldsymbol{\epsilon}}_{n+1}-\left.\frac{\partial \hat{\mathbf{G}}}{\partial \hat{\boldsymbol{\sigma}}}\right|_{n+1} ^{i+1} \Delta \hat{\boldsymbol{\lambda}}_{n+1}^{i+1}\right) \\
& \mathbf{r}_{n+1}^{\chi i+1}=\Delta \hat{\boldsymbol{\chi}}_{n+1}^{i+1}-\left.\left.\frac{\partial \hat{\boldsymbol{\chi}}}{\partial \hat{\boldsymbol{\epsilon}}^{p}}\right|_{n+1} ^{i+1} \frac{\partial \hat{\mathbf{G}}}{\partial \hat{\boldsymbol{\sigma}}}\right|_{n+1} ^{i+1} \Delta \hat{\boldsymbol{\lambda}}_{n+1}^{i+1}
\end{aligned}
$$

Solution of the system is then given by:

$$
\begin{gathered}
\mathbf{r}_{n+1}^{\sigma i+1}=\mathbf{r}_{n+1}^{\sigma i}+\left.\frac{\partial \mathbf{r}^{\sigma}}{\partial \hat{\boldsymbol{\sigma}}}\right|_{n+1} ^{i} \mathrm{~d} \hat{\boldsymbol{\sigma}}_{n+1}^{i+1}+\left.\frac{\partial \mathbf{r}^{\sigma}}{\partial \hat{\chi}}\right|_{n+1} ^{i} \mathrm{~d} \hat{\chi}_{n+1}^{i+1}+\left.\frac{\partial \mathbf{r}^{\sigma}}{\partial \hat{\boldsymbol{\lambda}}}\right|_{n+1} ^{i} \mathrm{~d} \hat{\lambda}_{n+1}^{i+1}=\mathbf{0} \\
\mathbf{r}_{n+1}^{\chi i+1}=\mathbf{r}_{n+1}^{\chi i}+\left.\frac{\partial \mathbf{r}^{\chi}}{\partial \hat{\boldsymbol{\sigma}}}\right|_{n+1} ^{i} \mathrm{~d} \hat{\boldsymbol{\sigma}}_{n+1}^{i+1}+\left.\frac{\partial \mathbf{r}^{\chi}}{\partial \hat{\chi}}\right|_{n+1} ^{i} \mathrm{~d} \hat{\boldsymbol{\chi}}_{n+1}^{i+1}+\left.\frac{\partial \mathbf{r}^{\chi}}{\partial \boldsymbol{\lambda}}\right|_{n+1} ^{i} \mathrm{~d} \hat{\lambda}_{n+1}^{i+1}=\mathbf{0} \\
\Delta \hat{\lambda}_{n+1}^{\beta i+1} \hat{\mathcal{F}}_{n+1}^{\beta i+1}=\Delta \hat{\lambda}_{n+1}^{\beta i} \hat{\mathcal{F}}_{n+1}^{\beta i}+\left.\frac{\partial \Delta \hat{\lambda}^{\beta} \hat{\mathcal{F}}^{\beta}}{\partial \hat{\boldsymbol{\sigma}}^{i}}\right|_{n+1} ^{i} \mathrm{~d} \hat{\boldsymbol{\sigma}}_{n+1}^{i+1}+\left.\frac{\partial \Delta \hat{\lambda}^{\beta} \hat{\mathcal{F}}^{\beta}}{\partial \hat{\boldsymbol{\chi}}^{i}}\right|_{n+1} ^{i} \mathrm{~d} \hat{\boldsymbol{\chi}}_{n+1}^{i+1} \\
+\left.\frac{\partial \Delta \hat{\lambda}^{\beta} \hat{\mathcal{F}}^{\beta}}{\partial \hat{\boldsymbol{\lambda}}^{i+1}}\right|_{n+1} ^{i} \mathrm{~d} \hat{\lambda}_{n+1}^{i+1}=0 \quad \beta=\text { LC, SI }
\end{gathered}
$$


Partial derivatives of $\mathbf{r}^{\sigma}$ and $\mathbf{r}^{x}$ are given by:

$$
\begin{aligned}
& \frac{\partial \mathbf{r}^{\sigma}}{\partial \hat{\boldsymbol{\sigma}}}=\mathbf{I}+\sum_{\beta=\mathrm{LC}, \mathrm{SI}} \Delta \hat{\lambda}^{\beta} \hat{\mathbf{D}}_{n} \frac{\partial^{2} \hat{\mathcal{G}}^{\beta}}{\partial \hat{\boldsymbol{\sigma}}^{2}} \\
& \frac{\partial \mathbf{r}^{\sigma}}{\partial \boldsymbol{\chi}}=\sum_{\beta=\mathrm{LC}, \mathrm{SI}} \Delta \hat{\lambda}^{\beta} \hat{\mathbf{D}}_{n} \frac{\partial^{2} \hat{\mathcal{G}}^{\beta}}{\partial \hat{\boldsymbol{\sigma}} \partial \hat{\boldsymbol{\chi}}} \\
& \frac{\partial \mathbf{r}^{\sigma}}{\partial \boldsymbol{\lambda}}=\hat{\mathbf{D}}_{n} \frac{\partial \hat{\mathbf{G}}}{\partial \hat{\boldsymbol{\sigma}}} \\
& \frac{\partial \mathbf{r}^{x}}{\partial \hat{\boldsymbol{\sigma}}}=-\sum_{\beta=\mathrm{LC}, \mathrm{SI}} \Delta \hat{\lambda}^{\beta} \frac{\partial \hat{\boldsymbol{\chi}}}{\partial \hat{\boldsymbol{\epsilon}}^{p}} \frac{\partial \hat{\mathcal{G}}^{\beta}}{\partial \hat{\boldsymbol{\sigma}}^{2}} \\
& \frac{\partial \mathbf{r}^{\chi}}{\partial \hat{\boldsymbol{\chi}}}=\mathbf{I}-\sum_{\beta=\mathrm{LC}, \mathrm{SI}} \Delta \hat{\lambda}^{\beta} \frac{\partial \hat{\boldsymbol{\chi}}}{\partial \hat{\boldsymbol{\epsilon}}^{p}} \frac{\partial^{2} \hat{\mathcal{G}}^{\beta}}{\partial \hat{\boldsymbol{\sigma}} \partial \hat{\boldsymbol{\chi}}} \\
& \frac{\partial \mathbf{r}^{\chi}}{\partial \boldsymbol{\lambda}}=-\frac{\partial \hat{\boldsymbol{\chi}}}{\partial \hat{\boldsymbol{\epsilon}}} \frac{\partial \hat{\mathbf{G}}}{\partial \hat{\boldsymbol{\sigma}}}
\end{aligned}
$$

where $\mathbf{I}$ is the identity matrix.

Newton-Raphson procedure of the Kuhn-Tucker condition can be written as:

$$
\begin{aligned}
\left.\Delta \hat{\lambda}_{n+1}^{\beta i} \frac{\partial \hat{\mathcal{F}}^{\beta}}{\partial \hat{\boldsymbol{\sigma}}^{i}}\right|_{n+1} ^{i} \mathrm{~d} \hat{\boldsymbol{\sigma}}_{n+1}^{i+1} & +\left.\Delta \hat{\lambda}_{n+1}^{\beta i} \frac{\partial \hat{\mathcal{F}}^{\beta}}{\partial \hat{\boldsymbol{\chi}}}\right|_{n+1} ^{i} \mathrm{~d} \hat{\boldsymbol{\chi}}_{n+1}^{i+1}+\hat{\mathcal{F}}_{n+1}^{\beta i} \mathrm{~d} \hat{\lambda}_{n+1}^{\beta i+1} \\
& =-\Delta \hat{\lambda}_{n+1}^{\beta i} \hat{\mathcal{F}}_{n+1}^{\beta i} \quad \beta=\text { LC, SI }
\end{aligned}
$$

since

$$
\left.\frac{\partial \hat{\mathcal{F}}^{\beta}}{\partial \hat{\boldsymbol{\lambda}}}\right|_{n+1} ^{i}=\mathbf{0} \quad \beta=\mathrm{LC}, \mathrm{SI}
$$

Restrictions $\Delta \hat{\lambda}^{\beta} \geqslant 0$ and $\hat{\mathcal{F}}^{\gamma} \leqslant 0$ are imposed by introducing two variables $\hat{\mu}^{\beta}$ and $\hat{\phi}^{\gamma}$ such as:

$$
\begin{aligned}
& \Delta \hat{\mu}^{\beta}=\pi^{\beta} \Delta \hat{\lambda}^{\beta} \quad \text { with } \pi^{\beta}=1 \text { if } \Delta \hat{\lambda}^{\beta} \geqslant 0 \text { and } \pi^{\beta}=0 \text { if } \Delta \hat{\lambda}^{\beta}<0 \\
& \hat{\phi}^{\gamma}=\rho^{\gamma} \hat{\mathcal{F}}^{\gamma} \quad \text { with } \quad \rho^{\gamma}=1 \text { if } \hat{\mathcal{F}}^{\gamma} \leqslant 0 \quad \text { and } \rho^{\gamma}=0 \text { if } \hat{\mathcal{F}}^{\gamma}>0
\end{aligned}
$$


When yield surface $\beta$ is found inactive, $\Delta \hat{\mu}^{\beta}=0$ and $\hat{\phi}^{\beta} \leqslant 0$. In this case, all the terms of $\beta$ row of Eq. (A12) are equal to 0 except $\hat{\mathcal{F}}_{n+1}^{\beta i}$ which remains as a non null pivot in the Jacobian matrix and fixes $\delta \lambda_{n+1}^{\beta i+1}=0$. This procedure gives an automatic update of the set of active yield surfaces in accordance to the condition given by Simo et al. (1988) that the consistency parameter $\Delta \hat{\lambda}^{\beta}$ associated to the active surface must be positive. For neutral loading $\left(\Delta \hat{\mu}^{\beta}=\Delta \hat{\lambda}^{\beta}=0\right.$ and $\left.\hat{\phi}^{\beta}=\hat{\mathcal{F}}^{\beta}=0\right)$, row and column corresponding to unknown $\Delta \hat{\lambda}^{\beta}$ are cancelled and a subsystem is solved.

\section{References}

Alawaji, H., Runesson, K., Sture, S., 1990. Integration of constitutive equations in soil plasticity. J. Engrg. Mech. 117 (8), 1771-1790.

Alawaji, H., Runesson, K., Sture, S., 1992. Implicit integration in soil plasticity under mixed control for drained and undrained response. Int. J. Num. Ana. Methods Geomech. 16 (10), 737-756.

Alcoverro, J., Gens, A. \& Alonso, E.E. 1998. Constitutive models for unsaturated soils. Thermodynamic approach. In: Proceedings of the 2nd International Conference on Unsaturated Soils, Int. Academic Pub., Beijing, pp. 455-460.

Alonso, E.E., Gens, A., Josa, A., 1990. A constitutive model for partially saturated soils. Géotechnique 40 (3), 405-430.

Bishop, A.W., Donald, I.B., 1961. The experimental study of partly saturated soils in the triaxial apparatus. In: Proceedings of the 5th International Conference on Soil Mechanics and Foundation Engineering, Paris: Dunod pp. 13-21.

Bolzon, G., Schrefler, B.A., Zienkiewicz, O.C., 1996. Elasto-plastic soil constitutive laws generalised to partially saturated states. Géotechnique 46 (2), 279-290.

Borja, R.I., Lee, S.R., 1990. Cam-clay plasticity, part I: implicit integration of elasto-plastic constitutive relations. Comput. Methods Appl. Mech. Engrg. 78 (1), 49-72.

Borja, R.I., 1991. Cam-clay plasticity, part II: implicit integration of constitutive equation based on a nonlinear elastic stress predictor. Comput. Methods Appl. Mech. Engrg. 88 (2), 225-240.

Chaboche, J.L., Cailletaud, G., 1996. Integration methods for complex plastic constitutive equations. Comp. Methods Appl. Mech. Engrg. 133 (1-2), 125-155.

Chateau, X., Dormieux, L., 1995. Homoginization of a non-saturated porous medium: Hill's lemma and applications. C.R.A.S. Paris, 320, série II b, pp. 627-634.

Coussy, O., 1995. Mechanics of Porous Continua. John Wiley and Sons, Chichester.

Crisfield, M.A., 1991. Non-finear Finite Element Analysis of Solids and Structures. John Wiley and Sons, Chichester.

Cui, Y.J., Delage, P., 1996. Yielding and plastic behaviour of an unsaturated compacted silt. Géotechnique 46 (2), 291-311.

Fredlund, D.G., Morgenstern, N.R., 1977. Stress state variables for unsaturated soils. J. Geotech. Engng. Div., Am. Soc. Civ. Engrs. 103 (GT5), 447-466.

Gens, A., Potts, D.M., 1988. Critical state models in computational geomechanics. Eng. Comp. 5 (3), 178-197.

Hassanizadeh, M., Gray, W.G., 1979a. General conservation equations for multiphase systems: 1, averaging procedure. Adv. Water Resources 2, 131-144.

Hassanizadeh, M., Gray, W.G., 1979b. General conservation equations for multiphase systems: 2, mass, momenta, energy and entropy equations. Adv. Water Resources 2, 191-203.

Hassanizadeh, M., Gray, W.G., 1980. General conservation equations for multiphase systems: 3, constitutive theory for porous media flow. Adv. Water Resources 2, 131-144.

Hassanizadeh, M., Gray, W.G., 1990. Mechanics and thermodynamics of multiphase flow in porous media including interphase boundaries. Adv. Water Resources 13 (4), 169-185.

Klinsinski, M., Mróz, Z., Runesson, R., 1992. Structure of constitutive equations in plasticity for different choices of state and control variables. Int. J. Plasticity 8 (3), 221-243. 
Ledesma, A., Chan, A.H.C, Vaunat, J., Gens, A., 1995. Finite element formulation of an elasto-plastic model for partially saturated soils. In: Owen, D.R.J., Oñate, E. (Eds.), Proceedings of the 4th International Conference on Computational Plasticity. Pineridge Press, Swansea, pp. 1677-1688.

Lewis, R.W., Schrefler, B.A., 1998. The Finite Element Method in the Static and Dynamic Deformation and Consolidation of Porous Media, 2nd Edition. John Wiley and Sons, Chichester.

Li, X.L., Charlier, R., 1995. A unified formulation of constitutive relations on saturated and partially saturated soils. In: Pande, G.N., Pietruszczak, S. (Eds.), Proceedings of the 5th International Conference on Numerical Models in Geomechanics. Balkema, Rotterdam, pp. 113-118.

Maâtouk, A., Leroueil, S., La Rochelle, P., 1995. Yielding and critical state of a collapsible unsaturated silty soil. Géotechnique 45 (3), 465-477.

Matyas, E.L., Radbakrishna, H.S., 1968. Volume change characteristics of partially saturated soils. Géotechnique 18, 432-448.

Mróz, Z., Rodzik, P., 1995. On the control of deformation process by plastic strain. Int. J. Plasticity 11 (7), 827-842.

Murad, M.A. 1995. A three-scale thermodynamical model of hydration swelling in smectite clays. Technical Report, Laboratório Nacional de Computaçao Científica, Rio de Janeiro.

Olivella, S., 1995. Nonisothermal multiphase flow of brine and gas through saline media. Ph.D. thesis. Universitat Politècnica de Catalunya, Barcelona.

Ohmaki, S., 1982. Stress-strain behaviour of anisotropically, normally consolidated cohesive soil. In: Dungar, R., Pande, G.N., Studer, J.A. (Eds.), Proceedings of the 1st International Symposium on Numerical Models in Geomechanics. Balkema, Rotterdam, pp. 250-269.

Ortiz, M., Simo, J.C., 1986. An analysis of a new class of integration algorithms for elastoplastic constitutive relations. Int. J. Num. Methods Engrg. 23 (3), 353-366.

Roscoe, K.H., Burland, J.B., 1968. On the generalized stress-strain behaviour of the 'wet' clay. In: Heyman, J., Leckic, F.A. (Eds.), Engineering Plasticity. Cambridge University Press, Cambridge, pp. 535609.

Runesson, K., 1987. Implicit integration of elastoplastic relations with reference to soils. Int. J. Num. Ana. Methods Geomech. 11 (3), 315-321.

Schrefler, B.A., Zhan Xiaoyong, 1993. A fully coupled model for water flow and air flow in deformable porous media. Water Resources Research 29 (1), 155-167.

Simo, J.C., Kennedy, J.G., Godvindjee, S., 1988. Non-smooth multisurface plasticity and viscoplasticity. Loading/unloading conditions and numerical algorithms. Int. J. Num. Meth. Engng. 26 (10), 21612185 .

Simo, J.C., Taylor, R.L., 1985. Consistent tangent operator for rate-independent elastoplasticity. Comp. Methods Appl. Mech. Engrg. 48, 101-118.

Sloan, S.W., 1987. Substepping schemes for the numerical integration of elastoplastic stress-strain relations. Int J Num Methods Engrg 24 (5), 893-911.

Thomas, H.R., He, Y., 1995. Analysis of coupled heat moisture and air transfer in a deformable unsaturated soil. Géotechnique 45 (4), 677-689.

Vicol, T., 1990. Comportement hydraulique et mécanique d'un sol fin non saturé: application à la modélisation. Thése de doctorat. Ecole Nationale des Ponts et Chaussées, Paris.

Wheeler, S.J., Sivakumar, V., 1995. A elasto-plastic critical state framework for unsaturated soil. Géotechnique 45 (1), 35-53.

Wheeler, S.J., 1996. Inclusion of specific water volume within an elasto-plastic model for unsaturated soil. Can. Geotech. J. 33 (1), 42-57.

Wood, D.M., 1990. Soil behaviour and critical state soil mechanics. Cambridge University Press, Cambridge. 